\title{
Reliability of transient elastography for the diagnosis of advanced fibrosis in chronic hepatitis C
}

\author{
U Arena, ${ }^{1}$ F Vizzutti, ${ }^{1}$ J G Abraldes, ${ }^{2}$ G Corti, ${ }^{3}$ C Stasi, ${ }^{3}$ S Moscarella, ${ }^{1}$ S Milani, ${ }^{4,5}$ \\ E Lorefice, ${ }^{4}$ A Petrarca, ${ }^{1}$ R G Romanelli, ${ }^{1}$ G Laffi, ${ }^{1}$ J Bosch, ${ }^{2}$ F Marra, ${ }^{1,5}$ M Pinzani ${ }^{1,5}$
}

${ }^{1}$ Department of Internal Medicine, University of Firenze, Firenze, Italy: ${ }^{2}$ Hepatic Hemodynamic Laboratory, Hospital Clinic, University of Barcelona, Barcelona, Spain;

${ }^{3}$ Unit for Infective and Tropical Diseases, University of Firenze, Firenze, Italy; ${ }^{4}$ Department of Clinical Pathophysiology, University of Firenze, Firenze, Italy; ${ }^{5}$ Center for Research, Higher Education and Transfer DENOThE, University of Firenze, Firenze, Italy

Correspondence to:

Professor M Pinzani,

Dipartimento di Medicina

Interna, Viale G.B. Morgagni, 85,

50134 Firenze, Italy;

m.pinzani@dmi.unifi.it

UA and FV contributed equally to this study.

Revised 13 March 2008 Accepted 15 April 2008 Published Online First 6 May 2008

\begin{abstract}
Background: Transient elastography (TE) has received increasing attention as a means to evaluate disease progression in patients with chronic liver disease.
\end{abstract}

Aim: To assess the value of TE for predicting the stage of fibrosis.

Methods: Liver biopsy and TE were performed in 150 consecutive patients with chronic hepatitis C-related hepatitis (92 men and 58 women, age 50.6 (SD 12.5) years on the same day. Necro-inflammatory activity and the degree of steatosis at biopsy were also evaluated.

Results: The areas under the curve for the prediction of significant fibrosis ( $\geqslant F 2$ ), advanced fibrosis ( $\geqslant F 3$ ) or cirrhosis were $0.91,0.99$ and 0.98 , respectively. Calculation of multilevel likelihood ratios showed that values of $\mathrm{TE}<6$ or $\geqslant 12,<9$ or $\geqslant 12$, and $<12$ or $\geqslant 18$, clearly indicated the absence or presence of significant fibrosis, advanced fibrosis, and cirrhosis, respectively. Intermediate values could not be reliably associated with the absence or presence of the target condition. The presence of inflammation significantly affected TE measurements in patients who did not have cirrhosis $(p<0.0001)$, even after adjusting for the stage of fibrosis. Importantly, TE measurements were not influenced by the degree of steatosis.

Conclusions: TE is more suitable for the identification of patients with advanced fibrosis than of those with cirrhosis or significant fibrosis. In patients in whom likelihood ratios are not optimal and do not provide a reliable indication of the disease stage, liver biopsy should be considered when clinically indicated. Necro-inflammatory activity, but not steatosis, strongly and independently influences TE measurement in patients who do not have cirrhosis.

In recent years a major effort has been directed at the identification of non-invasive methodologies for the assessment of the fibrotic progression of chronic liver diseases (CLDs) and for the longitudinal follow-up of patients, particularly following treatment.

A non-invasive medical device based on transient elastography (TE) (Fibroscan; Echosens, Paris, France) has received increasing attention. This system has been proposed for the measurement of liver stiffness, considered as a direct consequence of the fibrotic evolution of CLD. Accordingly, several studies aimed at evaluating the clinical usefulness and diagnostic accuracy of TE in predicting the degree of liver fibrosis have been published. ${ }^{1-10}$ Most of the published studies were based either on retrospective analysis or inadequate study designs, ie, liver biopsy not performed at the same time of TE and/or with suboptimal sample characteristics $^{2358}$ or the inclusion of different aetiological groups within the same analysis. ${ }^{6-10}$ Another problem emerging from the published reports concerns the different spectrum of fibrosis stages ("spectrum bias"; ie, over-representation of extreme stages of fibrosis) that make some assumptions unsuitable for clinical practice. ${ }^{3} 810$ Finally, it is increasingly evident that features of CLD other than fibrosis, ie, inflammatory infiltration, tissue necrosis or oedema, influence TE. ${ }^{9-12}$ Regardless, a key feature of the studies published so far is the dichotomisation of TE measurements by cut-off values selected by binary measures like sensitivity and specificity. However, it does not seem sufficiently informative to discriminate the optimal cut-off value in a wide range of TE values. It is therefore clinically relevant to pursue a more efficient approach that allows the exploration of the whole spectrum of TE measurements, such as the analysis of multilevel likelihood ratios (LRs). ${ }^{13}$

The purpose of the present study was to further assess the accuracy of TE in identifying hepatitis $C$ virus (HCV)-related CLD patients characterised by significant ( $\geqslant F 2$ ) or advanced ( $\geqslant F 3$ ) fibrosis, or cirrhosis (F4) by a multilevel LR analysis approach, and to evaluate the possible interfering role of inflammation and steatosis in a cohort of consecutive patients with HCV admitted to our centre for the histopathological assessment of disease progression.

\section{PATIENTS AND METHODS \\ Patients}

Between 1 September 2006 and 1 July 2007, 161 consecutive patients with HCV-related CLD (92 men and 58 women, age 50.6 (SD 12.5), age range 21-70 years) were referred to Azienda Ospedaliero Universitaria Careggi (AOUC) for the histopathological assessment of disease progression. On the same day as the liver biopsy, patients underwent an ultrasound examination of the complete upper abdomen and measurement of TE. Inclusion criteria were levels of serum alanine aminotransferase (ALT) $>1.5$-fold the upper normal limit, either persistently or intermittently, and detectable HCV RNA. Exclusion criteria were a body mass index of $30 \mathrm{~kg} / \mathrm{m}^{2}$ or greater, the presence of ascites at clinical or ultrasound examinations, the presence of hepatocellular carcinoma or previous/current clinical decompensation of the disease, co-infection with human immunodeficiency virus (HIV) or hepatitis $B$ virus (HBV), the use of intravenous drugs, previous or current alcohol abuse or the use of hepatotoxic drugs, genetic liver disease, 
Table 1 Clinical and laboratory findings in the study population

\begin{tabular}{|c|c|c|c|c|c|c|}
\hline Variable & Fo $(n=34)$ & $F 1(n=32)$ & $F 2(n=28)$ & F3 $(n=27)$ & $F 4(n=29)$ & p Value (ANOVA) \\
\hline Age (years) & 45.06 (SD 13.33) & 45.78 (SD 12.76) & 52.11 (SD 11.79) & 54.11 (SD 10.70)* & $57.69(\mathrm{SD} 8.64) \dagger$ & $<0.0001$ \\
\hline Male gender, $\mathrm{n}$ & $20(59 \%)$ & $17(53 \%)$ & $20(71 \%)$ & $19(70 \%)$ & $17(59 \%)$ & $0.53 \dagger$ \\
\hline BMI $\left(\mathrm{kg} / \mathrm{m}^{2}\right)$ & 23.8 (SD 3) & 23.6 (SD 3.2) & 23 (SD 2.1) & 24 (SD 2.9) & 22.9 (SD 2.8) & 0.33 \\
\hline Albumin $(\mathrm{g} / \mathrm{dl})$ & 4.12 (SD 0.45) & 4.29 (SD 0.25) & 4.27 (SD 0.38) & 4.02 (SD 0.29) & 3.64 (SD 0.65$) \$$ & $<0.0001$ \\
\hline INR & 0.98 (SD 0.05) & 0.99 (SD 0.08) & 0.99 (SD 0.06) & 0.99 (SD 0.06) & 1.16 (SD 0.24)§ & $<0.0001$ \\
\hline ALT (U/) & 74.79 (SD 61.58) & 100.91 (SD 104.54) & 92.61 (SD 111.40) & 157.22 (SD 140.17)* & 94.07 (SD 65.77) & 0.02 \\
\hline HCV genotype $3, n$ & $5(15 \%)$ & $4(13 \%)$ & $3(11 \%)$ & $4(15 \%)$ & $4(14 \%)$ & $0.61 \uparrow \dagger$ \\
\hline TE (kPa) & 5.6 (SD 1.8) & 6.8 (SD 2.3) & 8 (SD 1.6) & 13.9 (SD 2.9)** & 27.9 (SD 13.5)§ & $<0.0001$ \\
\hline
\end{tabular}

${ }^{*} p<0.05$ vs F0; $\dagger p<0.05$ vs F0 and F1. $t p<0.05$ vs F0, F1 and F2; and $p=0.05$ vs F3; $\$ p<0.05$ vs F0, F1, F2 and F3; $9 p<0.05$ vs F0, F1 and F2;

${ }^{* *} \mathrm{p}<0.05$ vs FO, F1, F2 and F4; $\dagger \chi^{2}$.

ALT, alanine aminotransferase; BMI, body mass index; $\gamma$-GT, gamma glutamyl-transpeptidase; HCV, hepatitis C virus; INR, international normalised ratio; TE, transient elastography.

autoimmune hepatitis, vascular diseases of the liver, biliary tract disorders, ongoing or recent (within 1 year) therapy with antiviral agents, cardiac failure, age younger than 18 years or older than 70 years, and pregnancy.

Laboratory tests, including serum bilirubin, albumin levels, ALT levels, gamma-glutamyltranspeptidase $(\gamma$-GT) levels, international normalised ratio (INR), platelet count, and creatinine levels were determined in all patients at the time of hospital admission. In each patient, liver biopsy and liver stiffness measurement (LSM) were performed on the same day. The nature of the study was explained to the patients, who provided a priori written informed consent before the beginning of the study, in accordance with the principles of the Declaration of Helsinki (Edinburgh revision, 2000).

\section{Transient elastography}

Following ultrasound examination, TE was performed by using the FibroScan apparatus, which consists of a $3.5 \mathrm{MHz}$ ultrasound transducer probe mounted on the axis of a vibrator. Mild amplitude and low-frequency vibrations $(50 \mathrm{~Hz})$ are transmitted to the liver tissue, inducing an elastic shear wave that propagates through the underlying liver tissue. The velocity of the wave is directly related to tissue stiffness. With the patient lying in dorsal decubitus with the right arm in the maximal abduction, ultrasound previously identified an adequate section of liver tissue free of large vascular structures and gallbladder in the intercostal space on the right lobe, and a skin mark was made to guide the position of TE transducer. The tip of the FibroScan transducer was covered with a drop of gel and placed perpendicularly in the intercostal space. Moreover, under control time motion and A-mode the operator checked the correct position of the probe during TE examination. ${ }^{35}$ Stiffness was measured on a cylinder of hepatic tissue of $1 \mathrm{~cm}$ in diameter and $2-4 \mathrm{~cm}$ in length. The operator was a staff physician (AU) who had previously performed at least 100 determinations in patients with CLD. As previously described ${ }^{5}$ and as suggested by the provider of the instrumentation, we considered representative measurements of the median value of

Table 2 Histopathological and transient elastography findings in the study population

\begin{tabular}{|c|c|c|c|c|c|c|}
\hline \multicolumn{4}{|l|}{ METAVIR } & \multicolumn{3}{|c|}{ Steatosis } \\
\hline Stage, n (\%) & Grade & & n (\%) & Score & & n (\%) \\
\hline \multirow[t]{4}{*}{ F0, 34 (22.67) } & $A$ & 0 & $8(23.53)$ & $S$ & 0 & $24(70.59)$ \\
\hline & & 1 & $22(64.71)$ & & 1 & $8(23.53)$ \\
\hline & & 2 & 4 (11.76) & & 2 & $1(2.94)$ \\
\hline & & 3 & $0(0)$ & & 3 & $1(2.94)$ \\
\hline \multirow[t]{4}{*}{$\mathrm{F} 1,32$ (21.33) } & $A$ & 0 & $7(21.88)$ & $S$ & 0 & $20(62.5)$ \\
\hline & & 1 & $14(43.75)$ & & 1 & $10(31.25)$ \\
\hline & & 2 & $11(34.37)$ & & 2 & $2(6.25)$ \\
\hline & & 3 & $0(0)$ & & 3 & $0(0)$ \\
\hline \multirow[t]{4}{*}{ F2, 28 (18.67) } & A & 0 & $2(7.14)$ & $S$ & 0 & $17(60.72)$ \\
\hline & & 1 & $16(57.15)$ & & 1 & $8(28.57)$ \\
\hline & & 2 & $10(35.71)$ & & 2 & $3(10.71)$ \\
\hline & & 3 & $0(0)$ & & 3 & $0(0)$ \\
\hline \multirow[t]{4}{*}{ F3, 27 (18) } & A & 0 & $0(0)$ & $S$ & 0 & $11(40.75)$ \\
\hline & & 1 & $8(27.58)$ & & 1 & $9(33.33)$ \\
\hline & & 2 & $17(58.62)$ & & 2 & $3(11.11)$ \\
\hline & & 3 & $4(13.80)$ & & 3 & $4(14.81)$ \\
\hline \multirow[t]{4}{*}{ F4, 29 (19.33) } & A & 0 & $0(0)$ & $S$ & 0 & $13(44.84)$ \\
\hline & & 1 & $8(27.58)$ & & 1 & $10(34.48)$ \\
\hline & & 2 & $17(58.62)$ & & 2 & $3(10.34)$ \\
\hline & & 3 & 4 (13.79) & & 3 & $3(10.34)$ \\
\hline
\end{tabular}

Fibrosis and necro-inflammatory activity were classified according to the METAVIR scoring system. Steatosis in liver specimens was arbitrarily classified by percentage of hepatocytes with fat deposits.

A, liver necro-inflammatory activity (grade); F, liver fibrosis (stage); n, number of patients; S, steatosis. 
ten successful acquisitions with a success rate of at least $60 \%$, and with an interquartile range (IOR) over a median ratio lower than $30 \%$.

\section{Ultrasound-assisted liver biopsy}

The clinical indication for biopsy was either to assess the evolution of a pre-diagnosed HCV-related liver disease, to confirm the nature of a suspected liver disease, or preliminary to a therapy with antiviral agents. Liver biopsy was performed on the right lobe of the liver with a 16 G semiautomatic modified Menghini needle system (BIOMOL; Hospital Service, Aprilia, Italy) under local anaesthesia and ultrasound guidance. Thirty minutes before the procedure, and after an overnight fast, patients received $5 \mathrm{mg}$ of diazepam and $5 \mathrm{mg}$ of atropine. Liver specimens were formalin-fixed and paraffin-embedded for histological evaluation. Sections of liver tissue, $5 \mu \mathrm{m}$ thick, were stained with haematoxylin \& eosin and Masson trichrome, and were examined by an experienced pathologist. The length of each liver specimen (in millimetres) was recorded. Only samples with a length $\geqslant 25 \mathrm{~mm}$ and including at least 11 complete portal tracts $^{14}$ were considered adequate for the purpose of the study. Histological diagnoses were established according to internationally accepted criteria. ${ }^{15}{ }^{16}$ Fibrosis (F) was staged on a five-point scale according to METAVIR: F0, no fibrosis; F1, portal fibrosis without septa; F2, portal fibrosis with few septa; F3, numerous septa without cirrhosis; and F4, cirrhosis. Necro-inflammatory activity (A) was also graded on a four-point scale according to METAVIR: A0, none; A1, mild; A2, moderate; and A3, severe. Steatosis in liver specimens was arbitrarily graded on a four-point scale: $0,<5 \% ; 1,5-24 \%$; $2,25-49 \%$; and $3, \geqslant 50 \%$ of hepatocytes with fat deposits.

\section{Statistical analysis}

The comparison between clinical and laboratory variables and TE values between fibrosis stages was conducted with ANOVA.

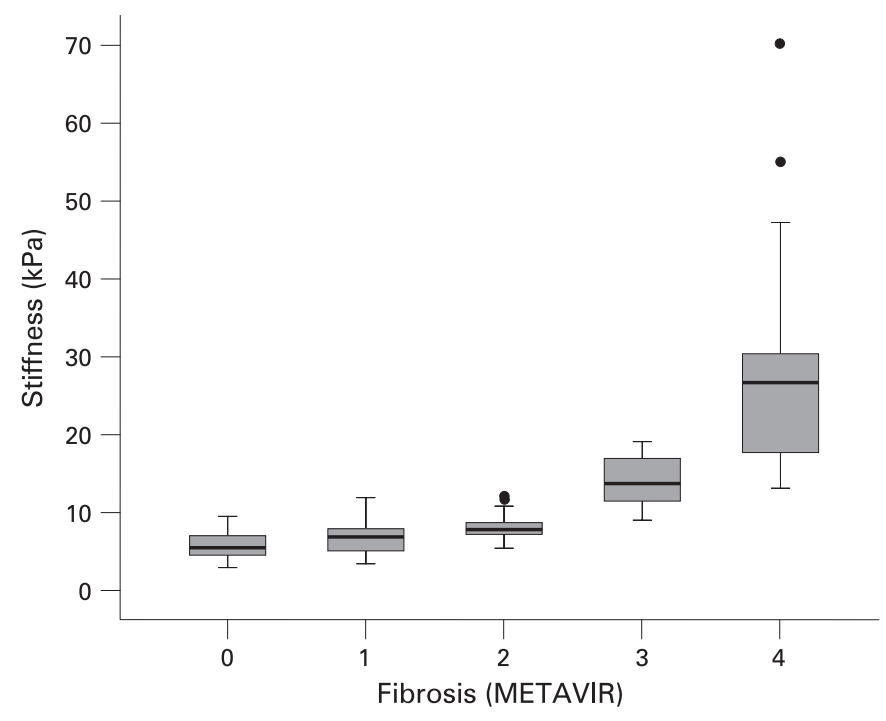

Figure 1 Box plots of transient elastography values in relation to the degree of fibrosis. Liver stiffness values are reported on the $y$-axis in kilopascals, and degree of fibrosis on the x-axis graded from 0 to 4 according to the METAVIR scoring system. The top and bottom of each box are first and second quartiles, respectively. The length of a box thus represents the interquartile range within which $50 \%$ of the values are located. The line through the each box represents the median. Error bars show minimum and maximum non-extreme values. $\bullet$, extreme values.

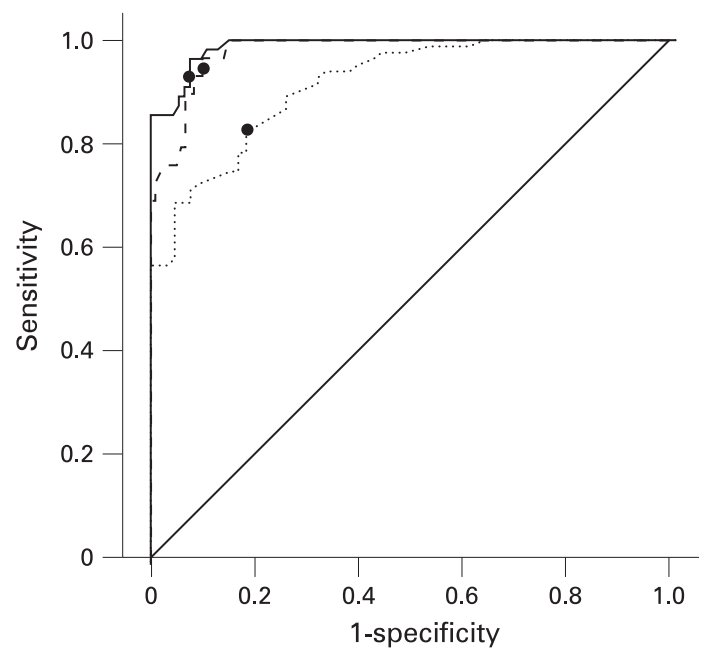

Figure 2 Receiver operating characteristic (ROC) curve showing the prediction of significant fibrosis (ie, METAVIR $\geqslant F 2$; dotted line), advanced fibrosis (ie, METAVIR $\geqslant F 3$; continuous line), and cirrhosis (ie, METAVIR F4; dashed line) with transient elastography (TE) in the whole patient population. The ideal area under the curve (AUC) is 1.00 . The straight line represents that based on chance alone ( $A U C=0.50$ ). The areas under the ROC curves were 0.91 ( $90 \%$ confidence interval $(\mathrm{Cl})$, 0.87 to 0.95$), 0.99(90 \% \mathrm{Cl}, 0.96$ to 0.99$)$, and $0.98(90 \% \mathrm{Cl}, 0.95$ to 0.99 ), respectively. Best possible cut-off values for each curve are indicated with black dots (see table 3). F, fibrosis (METAVIR stage).

Bonferroni analysis was used for post-hoc comparisons. The discriminative ability of $\mathrm{TE}$ to predict fibrosis $\geqslant \mathrm{F} 2, \geqslant \mathrm{~F} 3$ or cirrhosis (F4) was assessed by receiver operating characteristic (ROC) analysis. Multilevel LRs were used to explore the relationship between stiffness and fibrosis stage across the whole spectrum of TE values. The advantage of this approach is that, unlike sensitivity, specificity, and positive and negative predictive values, computation of LRs does not require dichotomisation of test results, which may discard useful diagnostic information. The likelihood ratio for each category was calculated by dividing the percentage of patients with the target condition ( $\geqslant F 2$, $\geqslant F 3$ or F4) in that category by the percentage without the condition in that category. Ninety per cent confidence intervals ${ }^{17}$ were calculated with the CIA software (Version 2.1.2). LRs above 10 and below 0.1 are considered to provide strong evidence to rule in or rule out diagnoses, respectively. ${ }^{13} 18$ The ability of clinical and laboratory variables to influence the diagnostic value of TE was assessed with multivariable logistic regression analysis. The relation between inflammation or steatosis and TE was adjusted by fibrosis stage in a multivariable linear regression analysis. All reported $p$ values are two-sided. Only $p$ values $\leqslant 0.05$ were considered statistically significant. Statistical analysis was performed with the SPSS 15.0 package.

\section{RESULTS}

\section{Characteristics of patients}

One hundred and fifty patients fulfilled the histopathological requirements and were considered for the statistical analysis (93\%). Eleven patients were excluded from the study because the liver biopsy length was $<25 \mathrm{~mm}$ and/or included fewer than 11 complete portal tracts. The major clinical and biochemical parameters of the patients included in the analysis are listed in table 1. Histopathological findings are reported in table 2. As shown in this table, the spectrum of fibrosis stages 
Table 3 Diagnostic accuracy of transient elastography

\begin{tabular}{llllllll}
\hline Stage & Cut-off & Sens & Spec & PPV & NPV & + LR & - LR \\
\hline$\geqslant$ F2 & 7.8 & 83 & 82 & 83 & 79 & 4.58 & 0.20 \\
$\geqslant$ F3 & 10.8 & 91 & 94 & 89 & 95 & 14.27 & 0.09 \\
F4 & 14.8 & 94 & 92 & 73 & 98 & 11.27 & 0.07 \\
\hline
\end{tabular}

The table presents the diagnostic accuracy of transient elastography (TE) in predicting significant fibrosis ( $\geqslant F 2$ ), advanced fibrosis $(\geqslant F 3)$, and cirrhosis (F4). Performance of the selected best TE cut-off values (in $\mathrm{kPa}$ ) are indicated.

$-\mathrm{LR}$, negative likelihood ratio; $+\mathrm{LR}$, positive likelihood ratio; NPV, negative predictive value; PPV, positive predictive value; Sens, sensitivity; Spec, specificity.

was homogeneously represented in our study population. The length of liver specimens was, on average, 33 (SD 0.7) mm, and included 15 (SD 3) complete portal tracts. All histological sections were considered adequate for evaluation with the METAVIR scoring system by the pathologist. Among patients with cirrhosis, endoscopy showed oesophageal varices in seven $(24 \%)$ and moderate portal hypertensive gastropathy in two (7\%). At the time of the study no patient with cirrhosis had previous or current clinical and/or ultrasonographic signs of clinical decompensation (ascites, encephalopathy, gastrointestinal bleeding, peripheral oedema). The mean duration of TE was 5 (SD 1.8) min. The mean number of measurements per patient was 12 (SD 1). The mean success rate and the mean IOR of TE measurements were $97.5 \%$ and $1.2 \mathrm{kPa}$, respectively. Figure 1 shows TE values for each stage of fibrosis.

\section{Accuracy of transient elastography for prediction of significant fibrosis ( $\geqslant$ F2)}

Figure 2 (dotted line) shows the accuracy of TE for the diagnosis of $\geqslant$ F2 fibrosis. The data-driven best cut-off for the diagnosis of significant fibrosis was $7.8 \mathrm{kPa}$ (table 3). Multilevel LRs (table 4) show that only values lower than $6 \mathrm{kPa}$ or equal to or greater than $12 \mathrm{kPa}$ were adequate to rule out or rule in significant fibrosis, respectively. Values between 6 and $12 \mathrm{kPa}$ could not reliably predict the presence or absence of significant fibrosis. None of the clinical or analytical variables considered was able to increase the predictive value of TE.

\section{Accuracy of transient elastography for prediction of advanced fibrosis $(\geqslant \mathrm{F} 3$ )}

The accuracy of TE for the diagnosis of $\geqslant F 3$ fibrosis is illustrated in fig 2 (continuous line). The data-driven best cutoff for the diagnosis advanced fibrosis was $10.8 \mathrm{kPa}$ (table 3). TE values lower than $9 \mathrm{kPa}$ or equal to or greater than $12 \mathrm{kPa}$ were adequate to rule out or rule in advanced fibrosis, respectively, as indicated by multilevel LRs (table 4). Multilevel LRs analysis show that values between 9 and $12 \mathrm{kPa}$ could not reliably predict the presence or absence of advanced fibrosis. None of the clinical or analytical variables considered was able to increase the predictive value of TE.

\section{Accuracy of transient elastography for prediction of cirrhosis (F4)}

Figure 2 (dashed line) shows the accuracy of TE for the diagnosis of cirrhosis. The data-driven best cut-off for the diagnosis cirrhosis was $14.8 \mathrm{kPa}$ (table 3). Multilevel LRs (table 4) show that values of less than $12 \mathrm{kPa}$ or equal to or greater than $18 \mathrm{kPa}$ were adequate to rule out or rule in cirrhosis, respectively. Values between 12 and $18 \mathrm{kPa}$ could not reliably predict the presence or absence of cirrhosis at multilevel LRs analysis. Among other clinical analytical variables, only platelet count marginally (although significantly) increased the predictive accuracy of TE for the diagnosis of cirrhosis. The area under the ROC (AUROC) curve for the model with TE and platelets was 0.98 ( $90 \% \mathrm{CI}, 0.97$ to 0.99 , ROC curve not shown).

\section{Relationship between necro-inflammatory activity and liver stiffness}

Necro-inflammatory activity at biopsy increased liver stiffness in each fibrosis stage, except for F4 patients (fig 3). On average, within each fibrosis stage in patients without cirrhosis but with A2-A3 had a liver stiffness $1.7 \mathrm{kPa}$ greater than patients with A1 (90\% CI, 0.971 to 2.460; $\mathrm{p}=0.0004)$ and $2.4 \mathrm{kPa}$ greater than patients with A0 (90\% CI, 1.396 to $3.463 ; p=0.0003)$. The degree of inflammation at biopsy could not be predicted from ALT levels. Unlike necro-inflammatory activity, regression analysis showed that ALT levels were not associated with stiffness.

\section{Relationship between steatosis and liver stiffness}

The degree of steatosis at biopsy did not influence TE measurements, even after adjusting for fibrosis stage $(p=0.35)($ fig 4$)$

\section{Complication related to the procedures}

No major complications were associated with percutaneous liver biopsy. Fifteen patients $(10 \%)$ experienced a self-limiting abdominal and/or right shoulder pain, and six patients (4\%) required a single dose of intravenous analgesic drug (tramadol). There were no complications associated with TE.

Table 4 Multilevel likelihood ratios (LRs) for the prediction of significant fibrosis, advanced fibrosis, and cirrhosis

\begin{tabular}{|c|c|c|c|c|c|}
\hline \multicolumn{2}{|c|}{ LRs for significant fibrosis $(\geqslant F 2)$} & \multicolumn{2}{|c|}{ LRs for advanced fibrosis $(\geqslant F 3)$} & \multicolumn{2}{|c|}{ LRs for cirrhosis (F4) } \\
\hline $\mathrm{TE}(\mathrm{kPa})$ & LR (CI) & TE (kPa) & LR (CI) & TE (kPa) & LRs (CI) \\
\hline$<6$ & $0.025(0.004$ to 0.139$)$ & $<6$ & $0(0$ to 0.190$)$ & $<12$ & 0 (0 to 0.139$)$ \\
\hline$\geqslant 6$ and $<9 *$ & $0.596(0.379$ to 0.931$)$ & $\geqslant 6$ and $<9$ & $0.034(0.006$ to 0.179$)$ & $\geqslant 12$ and $<15^{*}$ & $1.344(0.472$ to 3.831$)$ \\
\hline$\geqslant 9$ and $<12^{*}$ & 1.702 (0.712 to 4.159$)$ & $\geqslant 9$ and $<12^{*}$ & $0.979(0.415$ to 2.256$)$ & $\geqslant 15$ and $<18^{*}$ & $2.318(0.986$ to 5.449$)$ \\
\hline$\geqslant 12$ & Infinity (10.369 to infinity) & $\geqslant 12$ & Infinity (21.820 to infinity) & $\geqslant 18$ & $87.621(16.760$ to 458.074$)$ \\
\hline
\end{tabular}

Likelihood ratios above 10 and below 0.1 provide strong evidence to rule in or rule out diagnoses, respectively.

*Transient elastography (TE) value intervals that do not allow the prediction of the stage of fibrosis with a sufficient degree of confidence.

$\mathrm{Cl}$, confidence interval. 
Fo

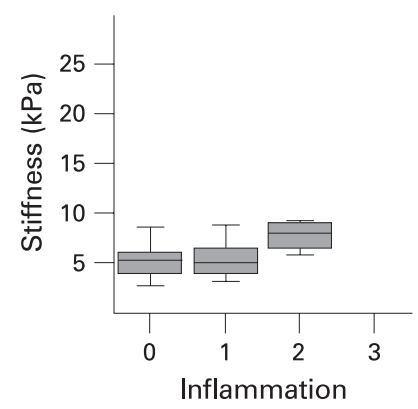

F1

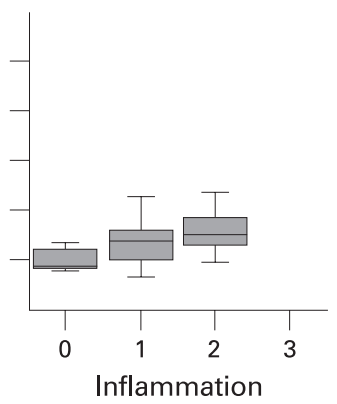

F2

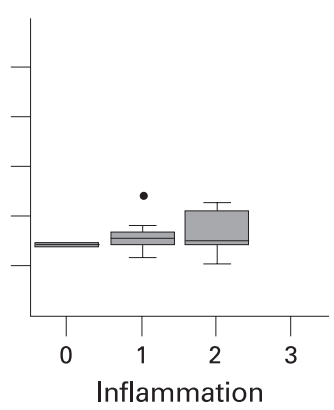

F3

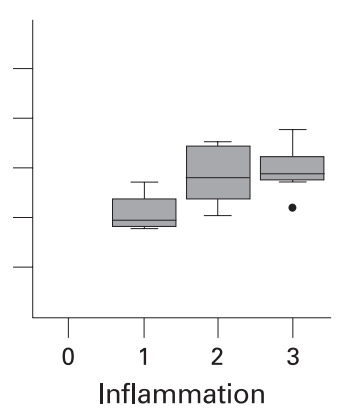

F4

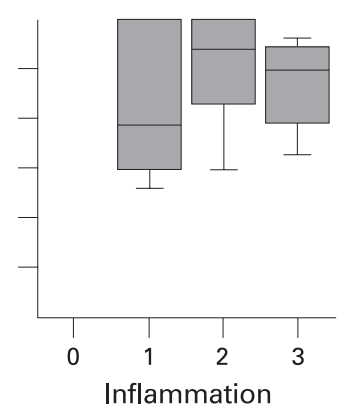

Figure 3 Box plots of transient elastography values in relation to the degree of necro-inflammatory activity. One graph for each degree of fibrosis $(F$, METAVIR scoring system) is provided. Liver stiffness values are reported on the y-axis, in kilopascals, and necro-inflammatory activity on the x-axis according to the METAVIR scoring system. The top and bottom of each box are first and second quartiles, respectively. The length of a box thus represents the interquartile range within which $50 \%$ of the values are located. The line through each box represents the median. Error bars show minimum and maximum non-extreme values. $F$, fibrosis (METAVIR stage). $\bullet$, extreme values.

\section{DISCUSSION}

In recent years much attention has been focused on whether non-invasive methodologies can detect significant or advanced liver fibrosis. At their present stage of development, all noninvasive methodologies have been shown to be effective in predicting the presence of either no or minimal disease or advanced disease, rather than being able to precisely assess the progression of fibrosis in a step-wise fashion. ${ }^{19}$ However, all the proposed approaches, used singly or in combination, are likely to provide a significant contribution to the diagnostic management of patients with CLD. TE is a promising clinical methodology for the rapid and non-invasive assessment of disease progression in viral and non-viral CLD, which is currently subjected to extensive validation. In this context, and based on the evidence reported so far, the present study was performed in order to provide a more definite indication of the real clinical utility of this methodology by employing a more rigorous study design, particularly concerning the use of liver biopsy as the reference standard, ${ }^{14}$ and a more informative statistical analysis of the data. ${ }^{13}$

The results of the present study indicate that, although TE is able to separate F3 and F4 from other stages of fibrosis, an important overlap is observed among patients with lower degrees of liver fibrosis (F0, F1 and F2). In addition, while TE mean values of patients with stages F3 and F4 were significantly different at Bonferroni analysis, an important overlap of TE values was also observed between these advanced stages of fibrosis. However, data reported in table 3 indicate that TE reliably predicts the presence of advanced fibrosis and cirrhosis.

To overcome the limitations emerging from the use of single cut-off values in clinical practice and to explore the whole spectrum of TE measurements, the analysis of multilevel LRs was introduced in order to estimate the likelihood of having a target degree of fibrosis according to TE measurements. Indeed, the use of multilevel LRs analysis is a more informative and correct approach since it explores the whole spectrum of TE measurement. ${ }^{13}{ }^{18}$ In the present study, TE values $<6,<9$ and $<12 \mathrm{kPa}$ strongly predict the absence of significant or advanced fibrosis and cirrhosis, respectively, whereas TE values $\geqslant 12$ and $\geqslant 18 \mathrm{kPa}$ strongly predict the presence of significant or advanced fibrosis and cirrhosis, respectively. The relative intermediate values do not appear adequate to rule out or to rule in the stage of fibrosis. Of note, no clinical or analytical variable considered in the present study concretely contributed to the predictive value of TE.

The high TE value $(\geqslant 18 \mathrm{kPa}$ ) requested to predict the presence of cirrhosis with an elevated level of confidence is likely due to the significant overlap existing between TE values obtained in F3 and F4 stages and to the difficulty of defining an appropriate level of fibrosis evolution beyond the F4 stage. These interpretative problems occur also when considering single cut-off values as reflected by the wide range of the reported $\mathrm{TE}$ values predicting the presence of cirrhosis (from 12.5 to $17.6 \mathrm{kPa}$ ). ${ }^{5}$ Accordingly, TE appears to be a methodology more suitable for predicting advanced fibrosis (ie, $\geqslant F 3$ ) rather than cirrhosis.
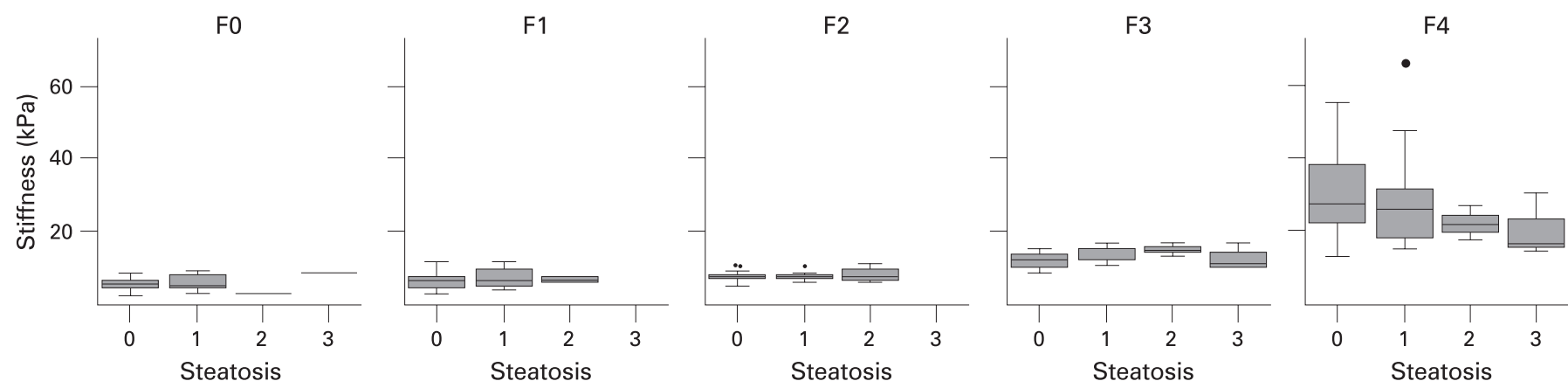

Figure 4 Box plots of transient elastography values in relation to the degree of steatosis. One graph for each degree of fibrosis (F, METAVIR scoring system) is provided. Liver stiffness values are reported on the $y$-axis in kilopascals, and degree of steatosis on the x-axis arbitrarily graded from 0 to 3 $(0,<5 \% ; 1,5-24 \% ; 2,25-49 \%$; and $3, \geqslant 50 \%$ of hepatocytes with fat deposits). The top and bottom of each box are first and second quartiles, respectively. The length of a box thus represents the interquartile range within which $50 \%$ of the values are located. The line through each box represents the median. Error bars show minimum and maximum non-extreme values. F, fibrosis (METAVIR stage). •, extreme values. 
An interesting finding of the present study is the demonstration that necro-inflammatory activity at biopsy influences TE values. Indeed, necro-inflammatory activity increases liver stiffness even after adjusting for fibrosis stage in patients who do not have cirrhosis. On average, within each fibrosis stage, patients without cirrhosis but with A2-A3 had a liver stiffness $1.7 \mathrm{kPa}$ greater than patients with $\mathrm{A} 1$, and $2.4 \mathrm{kPa}$ greater than patients with $\mathrm{A} 0$. These results confirm reports from literature suggesting the impact of necro-inflammatory activity on TE during aminotransferase flares, ${ }^{9}$ acute viral hepatitis, ${ }^{11}{ }^{12}$ in patients with miscellaneous $\mathrm{CLD},{ }^{10}$ and in patients who have received a liver transplant and who have recurrent hepatitis $C .{ }^{20}$ Therefore, the degree of necro-inflammatory activity may limit the diagnostic accuracy of TE especially in patients with lowerintermediate stages of fibrosis. This may account, at least in part, for the range of uncertainty observed in the multilevel LR analysis shown in table 4. As is evident, the high TE value emerging from the LR analysis for the prediction of significant fibrosis ( $\geqslant \mathrm{F} 2$ ), ie, $\geqslant 12 \mathrm{kPa}$, is identical to the value required for the diagnosis of advanced fibrosis ( $\geqslant F 3$ ). As a result, it seems reasonable to consider cautiously the TE values obtained in patients with aminotransferase flares or unstable biochemical activity. It is of note that in our study population the degree of inflammation at biopsy could be not predicted from ALT levels. Moreover, ALT levels were not associated with liver stiffness. Therefore, the influence of inflammation on stiffness could not be factorised by using ALT. These findings are in contrast with those of Coco et al, ${ }^{9}$ who reported an association between TE values and ALT levels. Of note, the cohort of patients investigated by these authors was not homogeneous in terms of aetiology, and patients with aminotransferases flares were included. Furthermore, in the study by Coco et al the relationship between necro-inflammation at histology, biochemical activity and TE values was prejudiced, at least in part, by the fact that biopsy was not simultaneously performed with TE.

This study also provides evidence on the possible interfering role of steatosis on TE measurements. In our study population the extent of steatosis at biopsy did not influence TE within each fibrosis stage. These results are consistent with those of Sandrin et $\mathrm{al}^{2}$ obtained in a smaller cohort of patients with HCV in whom liver biopsy was not performed simultaneously with TE, with the study by Ziol et $a l^{\beta}$ in which TE was performed within 6 months after liver biopsy, and with those by Kim et a ${ }^{21}$ attained in healthy potential liver donors in whom TE values were not correlated with steatosis. It must be pointed out that only a minority of patients had massive steatosis (eight patients with $\geqslant 50 \%$ of fatty hepatocytes) in our cohort. However, as recently reported by Yoneda et a ${ }^{22}$ the extent of steatosis does not seem to affect TE even in a cohort of patients with nonalcoholic fatty liver disease.

In summary, TE is more suitable for the identification of patients with advanced fibrosis rather than those with significant fibrosis or cirrhosis. In patients in whom LRs are not optimal for providing a reliable indication of the disease stage, liver biopsy should be considered when clinically indicated. Necro-inflammatory activity, but not steatosis, strongly and independently influences TE measurement in patients who do not have cirrhosis.
Acknowledgements: The authors thank the Unitat de Suport a la Estadística i Metodología (IDIBAPS, Barcelona) for statistical advice.

Funding: This work was funded by grants from the Italian MIUR, the University of Florence and the Italian Liver Foundation and Instituto de Salud Carlos III, Spain (FIS 05/0519 and FIS 06/0623).

Competing interests: None declared.

Ethics approval: The study protocol was approved by the Investigation and Ethics Committee of the Azienda Ospedaliero Universitaria di Careggi, Firenze, on 4 September 2006.

\section{REFERENCES}

1. Sandrin L, Tanter M, Gennisson JL, et al. Shear elasticity probe for soft tissues with $1 \mathrm{D}$ transient elastography. IEEE Trans Ultrason Ferroelectr Freq Control 2002;49:436-46.

2. Sandrin L, Fourquet B, Haquenoph JM, et al. Transient elastography: a new noninvasive method for assessment of hepatic fibrosis. Ultrasound Med Biol 2003;29:1705-13.

3. Ziol M, Handra-Luca A, Kettaneh A, et al. Non-invasive assessment of liver fibrosis by measurement of stiffness in patients with chronic hepatitis C. Hepatology 2005; 41:48-54.

4. Colletta C, Smirne C, Fabris C, et al. Value of two non invasive methods to detect progression of fibrosis among HCV carriers with normal aminotransferases. Hepatology 2005:42:838-45.

5. Castéra L, Vergniol J, Foucher J, et al. Prospective comparison of transient elastometry, fibrotest, APRI, and liver biopsy for the assessment of fibrosis in chronic hepatitis C. Gastroenterology 2005;128:343-50.

6. Foucher J, Chanteloup E, Vergniol J, et al. Diagnosis of cirrhosis by transient elastography (Fibroscan): a prospective study. Gut 2006;55:403-8.

7. de Lédinghen V, Douvin $C$, Kettaneh $A$, et al. Diagnosis of hepatic fibrosis and cirrhosis by transient elastography in HIV/hepatitis C virus co-infected patients. J Acquir Immune Defic Syndr 2006;41:175-9.

8. Ganne-Carrié $\mathbf{N}$, Ziol M, de Ledinghen $\mathbf{V}$, et al. Accuracy of liver stiffness measurement for the diagnosis of cirrhosis in patients with chronic liver disease. Hepatology 2006;44:1511-7.

9. Coco B, Olivieri F, Maina AM, et al. Transient elastography: a new surrogate marker of liver fibrosis influenced by major changes of aminotransferases. J Viral Hep 2006;14:360-9.

10. Fraquelli $\mathbf{M}$, Rigamonti $\mathbf{C}$, Casazza G, et al. Reproducibility of transient elastography in the evaluation of liver fibrosis in patients with chronic liver disease. Gut 2007:56:968-73.

11. Arena U, Vizzutti F, Corti G, et al. Acute viral hepatitis increases liver stiffness values measured by transient elastography. Hepatology 2008;47:380-4.

12. Sagir A, Erhardt A, Schmitt M, et al. Transient elastography is unreliable for detection of cirrhosis in patients with acute liver damage. Hepatology 2008; $47: 592-5$

13. Deeks JJ, Altman DG. Diagnostic tests 4: likelihood ratios. BMJ 2004;329:168-9.

14. Guido M, Rugge M. Liver fibrosis: natural history may be affected by the biopsy sample. Gut 2004;53:1878.

15. The French METAVIR Cooperative Study Group. Intraobserver and interobserver variations in liver biopsy interpretation in patients with chronic hepatitis C. Hepatology 1994;20:15-20.

16. Bedossa P, Poynard T. An algorithm for the grading of activity of chronic hepatitis $\mathbf{C}$. The METAVIR Cooperative Study Group. Hepatology 1996;24:289-93.

17. Sterne JA, Davey SG. Sifting the evidence - what's wrong with significance tests? BMJ 2001;322:226-31.

18. Guyatt G, Sackett DL, Haynes B. Evaluating diagnostic tests. In: Haynes B, Sackett DL, Guyatt G, Tulliez M, eds. Clinical epidemiology. 3rd edn. Philadelphia: Lippincott Williams \& Wilkins, 2006: 273-322.

19. Pinzani M, Vizzutti F, Arena U, et al. Technology insight: non-invasive assessment of liver fibrosis by biochemical scores and elastography. Nature Clin Prac Gastroenterol Hepatol 2008;5:95-106.

20. Rigamonti C, Donato MF, Fraquelli M, et al. Transient elastography predicts fibrosis progression in patients with recurrent hepatitis $\mathrm{C}$ after liver transplantation. Gut 2008;57:821-7.

21. Kim KM, Choi WB, Park SH, et al. Diagnosis of hepatic steatosis and fibrosis by transient elastography in asymptomatic healthy individuals: a prospective study of living related potential liver donors. J Gastroenterol 2007;42:382-8.

22. Yoneda M, Mawatari H, Fujita K, et al. Noninvasive assessment of liver fibrosis by measurement of stiffness in patients with nonalcoholic fatty liver disease (NAFLD). Dig Liver Dis (in press). 


\section{GUT}

\section{Reliability of transient elastography for the diagnosis of advanced fibrosis in chronic hepatitis C}

U Arena, F Vizzutti, J G Abraldes, et al.

Gut 2008 57: 1288-1293 originally published online April 30, 2008

doi: 10.1136/gut.2008.149708

Updated information and services can be found at:

http://gut.bmj.com/content/57/9/1288.full.html

These include:

References This article cites 20 articles, 6 of which can be accessed free at: http://gut.bmj.com/content/57/9/1288.full.html\#ref-list-1

Article cited in:

http://gut.bmj.com/content/57/9/1288.full.html\#related-urls

Email alerting service

Receive free email alerts when new articles cite this article. Sign up in the box at the top right corner of the online article.

Topic Articles on similar topics can be found in the following collections Collections

$$
\text { Hepatitis C (128 articles) }
$$

Notes

To request permissions go to:

http://group.bmj.com/group/rights-licensing/permissions

To order reprints go to:

http://journals.bmj.com/cgi/reprintform

To subscribe to BMJ go to:

http://group.bmj.com/subscribe/ 